\title{
Fatores de risco perinatais e avaliação neuropsicomotora em crianças pré-termo com e sem hemorragia peri-intraventricular
}

\author{
Perinatal risk factors and neuropsychomotor evaluation in preterm \\ infants, with and without peri intraventricular hemorrhage
}

\section{Maria Júlia Parcias do Rosário}

Claudia Maria de Lorenzo²

\section{Anelise Sonza ${ }^{3}$}

Silvia Rosane Parcias ${ }^{4}$

Endereço para Correspondência:

Anelise Sonza

Departamento de Fisioterapia, Universidade do Estado de Santa Catarina

Rua Pascoal Simone, 358, Bairro Coqueiros

90050-170 - Florianópolis, SC [Brasil]

anelise.sonza@udesc.br

\footnotetext{
1 Residência Médica Hospital de Clínicas de Porto Alegre HCPA. Porto Alegre, RS - Brasil.

ORCID: https://orcid.org/0000-0002-1655-9082

2 Hospital Infantil Joana de Gusmão - HIJG. Florianópolis, SC - Brasil.

ORCID: https://orcid.org/0000-0001-7554-289X

3 Departamento de Fisioterapia, Centro de Ciências da Saúde e do Esporte - CEFID, Universidade do Estado de Santa Catarina - UDESC. Florianópolis, SC - Brasil. ORCID: https://orcid.org/0000-0003-0056-4984

4 Departamento de Ciências Biológicas, Centro de Ciências da Saúde e do Esporte - CEFID, Universidade do Estado de Santa Catarina - UDESC. Florianópolis, SC - Brasil. ORCID: https://orcid.org/0000-0003-2030-2607
}

\begin{abstract}
Resumo
Introdução: A hemorragia peri-intraventricular (HPIV) é multifatorial e pode causar sequelas no desenvolvimento neuropsicomotor (DNPM) da criança. Objetivo: Analisar fatores de risco perinatais e o DNPM de crianças de alto risco nascidas pré-termo com e sem HPIV. Método: Realizou-se um estudo observacional caso-controle, com uma coorte clínica de um ambulatório de seguimento de prematuros de alto risco, com e sem HPIV. Foram utilizados questionários e o teste Denver II. Realizou-se análise bivariada pelo teste de Fischer e regressão logística multivariada $(p \leq 0,05)$. Resultados: Foram avaliadas 47 crianças prematuras de alto risco, sendo 14 com HPIV, e 33 sem. Idade gestacional (OR=16,5; $p<0,001)$, peso ao nascer $(\mathrm{OR}=9,23 ; p<0,01)$ e práticas neonatais (ventilação, intubação, etc.) $(\mathrm{OR}=6,68$; $p<0,01)$ estão relacionados com a HPIV. Não foi encontrada alteração do DNPM em crianças com e sem HPIV. Conclusões: A baixa idade gestacional, baixo peso ao nascer e práticas neonatais estão associadas à ocorrência de HPIV. Não há associação entre HPIV e o DNPM em prematuros.
\end{abstract}

Descritores: Fator de risco. Recém-nascido prematuro. Desenvolvimento infantil.

\begin{abstract}
Introduction: Peri-intraventricular hemorrhage (PIVH) is multifactorial and may cause disturbances in the child's neuropsychomotor development (NPMD). Objective: To analyze perinatal risk factors and NPMD of high-risk preterm infants with and without PIVH. Method: Observational case-control study, a clinical cohort was conducted in a follow-up outpatient clinic of high risk newborns. High risk preterm infants with and without PIVH were accessed. Questionnaires and the Denver II test were used. Bivariate analysis through Fischer test and multivariate logistic regression was performed ( $\mathrm{p} \leq 0.05)$. Results: 47 high risk preterm infants were evaluated, 14 with and 33 without PIVH history. Gestational age (OR $=16.5, \mathrm{p}<0.001)$, birth weight $(\mathrm{OR}=9.23, \mathrm{p}<0.01)$ and neonatal practices $(\mathrm{OR}=6.68, \mathrm{p}<0.01)$ are related to the HPIV. There was no association between NPMD and HPIV for this sample. Conclusions: Low gestational age, low birth weight and neonatal practices are associated with PIVH. No association between HPIV and NPMD was found in preterm infants.
\end{abstract} Keywords: Risk factors. Infant, Premature. Child development. 


\section{Introdução}

A hemorragia peri-intraventricular (HPIV) é uma afecção de causas multifatoriais, na qual estão envolvidos fatores vasculares, hemodinâmicos, inflamatórios e infecciosos, podendo causar sequelas neuropsicomotoras e levar à paralisia cerebral e/ou deficit cognitivo e comportamental ${ }^{1,2}$. É considerada uma importante causa de morbimortalidade, podendo ser associada à baixa idade gestacional e ao baixo peso ao nascimento ${ }^{1,3}$, sendo uma das principais afecções neurológicas que acometem o recém-nascido pré-termo (RNPT), principalmente aqueles de muito baixo peso ${ }^{1,3}$. Portanto, prematuridade, baixo peso ao nascer e índice de Apgar são apontados como fatores de risco perinatais e esses neonatos são considerados de alto risco ${ }^{4,5}$. Segundo a Organização Mundial da Saúde, um bebê recém-nascido pré-termo é aquele que apresenta idade gestacional menor que 37 semanas e menos de $2500 \mathrm{~g}^{6}$. A gravidade da hemorragia intracraniana em lactentes prematuros pode ser avaliada de acordo com a classificação de Papile et al. ${ }^{7}$, a qual é considerada como um dos sistemas mais utilizados. Essa classificação foi estabelecida com base na localização topográfica da hemorragia e na presença de dilatação ventricular, e pode variar dos graus I a IV, conforme a extensão da hemorragia. São fatores extra e intravasculares relacionados à ocorrência de hemorragia intraventricular: suporte vascular precário, atividade fibrinolítica aumentada, pressão tissular diminuída, flutuação, aumento e diminuição do fluxo sanguíneo cerebral ${ }^{5,8}$.

Destacam-se, como fatores de risco para maior disfunção neurológica, sepse em recém-nascidos no período neonatal com sepse9 ventilação mecânica ${ }^{10}$ e presença de HPIV III e $\mathrm{IV}^{11,12}$, baixo índice de Apgar ${ }^{13}$ e convulsões perinatais ${ }^{9}$, além do baixo nível socioeconômico materno ${ }^{4,14}$

O desenvolvimento neuropsicomotor de RNPT portadores de HPIV deve ser investigado e analisado, por ter importante repercussão no futuro desenvolvimento neurológico destas crianças $^{5}$, alterando sua qualidade de vida e também a dos seus cuidadores ${ }^{15}$. Ainda, alguns estudos ${ }^{3,14,16}$ apontam que o baixo peso ao nascer e a IG interferem no desenvolvimento neuropsicomotor (DNPM) da criança, gerando diferenças no desempenho escolar, na linguagem e no comportamento.

Observa-se que os prematuros internados podem apresentar atraso precoce no desenvolvimento motor, de forma grave ou limítrofe ${ }^{15}$, com movimentação anormal ou ausente na primeira semana, o que ocasiona alta incidência de anormalidade no exame neurológico ${ }^{17}$. Verifica-se também que atrasos neurológicos no RNPT e na criança com 1 ano de idade predizem alterações futuras nas habilidades neuromotoras, na função cognitiva e nos indicadores educacionais, comportamentais e psiquiátricos ${ }^{17}$. Portanto, identificar fatores de risco e acompanhar esses RN seriam as principais ações básicas para prevenir problemas futuros no desenvolvimento infantil $1^{11,12,15}$.

Baseado no exposto, objetivou-se neste estudo verificar os fatores de risco perinatais e avaliar o desenvolvimento neuropsicomotor de crianças prematuras, de alto risco (baixo peso ao nascer, prematuridade e baixo índice de Apgar), com e sem hemorragia peri-intraventricular.

\section{Métodos}

Trata-se um estudo observacional de delineamento transversal caso-controle associado a uma coorte clínica, realizado no Brasil, na cidade de Florianópolis, na área ambulatorial do Hospital Infantil Joana de Gusmão (HIJG), vinculada à Secretaria Estadual de Saúde de Santa Catarina - polo de referência estadual para as doenças de média e alta complexidade.

O projeto foi aprovado pelo Comitê de Ética e Pesquisa do Hospital Infantil Joana de Gusmão (HIJG), sob o número 715.562. Pais ou responsáveis pelas crianças participantes assinaram o Termo de Consentimento Livre e Esclarecido (TCLE). 
Foram incluídas no estudo crianças nascidas pré-termo, de alto risco, de 0 a 6 anos, com idade gestacional <37 semanas, com ou sem hemorragia peri-intraventricular, oriundas do HIJG ou encaminhadas a este, atendidas no ambulatório de seguimento de recém-nascidos de alto risco, durante o período de outubro a novembro de 2014. Foram excluídas da pesquisa crianças que foram a óbito durante o período de acompanhamento; as que o responsável negou sua participação no estudo; as que no dia da realização do teste apresentaram algum sintoma ou doença aguda impedindo-as de realizarem o teste ou dificultando a sua realização, além daquelas com doenças neurológicas que não a doença em estudo.

Para o cálculo do tamanho da amostra, utilizou-se o programa OPENEPI (Emory University, EUA), tanto para estudos de caso quanto para controle, com significância estatística de $5 \%$ de confiança $(\mathrm{p}<0,05)$, poder de $80 \%$, odds ratios (OR) de pelo menos o dobro $(\mathrm{OR}=2,0)$. Assim, uma amostra final de 14 casos com HPIV e 33 sem HPIV foi selecionada e calculada como suficiente para a pesquisa.

Para minimizar os possíveis vieses, os instrumentos de coleta dos dados foram aplicados por um só pesquisador, em horário e local semelhante e foi realizada seleção de casos e controles amostrados de uma mesma população, utilizando-se igual técnica para avaliação maturacional do desenvolvimento da criança. $\mathrm{O}$ pesquisador ainda aplicou questionários de informações gerais e da situação socioeconômica. Os dados pré e perinatais foram acessados por meio do prontuário eletrônico.

Como procedimentos de coleta dos dados, realizou-se o contato mediante entrevista com a mãe, durante a consulta de puericultura, para solicitação de participação da criança no estudo e esclarecimento sobre o correto preenchimento do questionário de identificação e coleta de dados socioeconômicos e sobre a aplicação dos testes. Após a concordância dos termos da pesquisa foi assinado o termo de consentimento livre e esclarecido pela mãe ou responsável legal.
A avaliação maturacional do desenvolvimento nos prematuros foi realizada durante a consulta ou agendada para data próxima e teve duração de aproximadamente 20 (vinte) minutos. Todos os registros tiveram a garantia de sigilo das respostas e da identidade dos participantes.

Os instrumentos de pesquisa selecionados foram: (1) questionário de informações gerais para caracterização da mãe (tipo de parto, realização de pré-natal e comorbidades); (2) situação socioeconômica, por meio do instrumento adotado pela Associação Nacional de Empresa de Pesquisa - Critério de Classificação Econômica Brasil ${ }^{18}$, o qual classifica a população em classes econômicas A1 e 2, B1 e 2, C1 e 2, D e E mediante pontos de corte de 46 a zero pontos sendo "A1" o maior poder aquisitivo; (3) dados pré e perinatais do prematuro (sexo; idade gestacional; peso ao nascer; existência de doenças neonatais; práticas neonatais associadas como ventilação, intubação, punção calcanhar, dentre outras; Apgar; presença e grau de hemorragia peri-intraventricular identificado pelo ultrassom transfontanelar - essas informações foram colhidas do prontuário eletrônico dos avaliados; (4) avaliação da condição atual do desenvolvimento maturacional da criança pelo teste de Denver II $^{19}$ (Denver Developmental Screening Test II), adaptado para o Português por Figueiras ${ }^{20}$, sendo composto de 125 itens divididos em quatro áreas, a saber: (1) as capacidades pessoais e sociais; (2) o desempenho motor fino adaptativo; (3) o desempenho motor grosso; e (4) a linguagem. A avaliação de Denver II é aplicada em torno de 20 minutos e detecta atrasos no desenvolvimento de meninos e meninas de 0 a 6 anos de idade. A criança deveria realizar as tarefas esperadas para o ponto de corte de ao menos $75 \%$ do esperado para a idade avaliada ${ }^{21}$.

As respostas foram codificadas em passa, falha, ou recusa (impossível de testar e explicar), de acordo com a habilidade da criança em realizar determinado item. O desempenho dos participantes analisados foi classificado segundo o número de falhas, sendo considerado o seguinte: (1) anormal, quando apresentou duas ou 
mais falhas independentemente da área ou setor; (2) questionável, quando obteve apenas uma falha; (3) normal, quando a criança avaliada não apresentou nenhuma falha ${ }^{22}$. O entrevistador foi previamente treinado na administração do teste de acordo com o manual de treinamento do teste original ${ }^{19}$. No momento da avaliação de Denver II, foi considerada a idade corrigida, ou seja, aquela que a criança teria se tivesse nascido de 40 semanas.

Os dados foram tabulados em uma planilha eletrônica no programa Excel $\mathrm{XP}^{\circledR}$ e então transportados para o programa SPSS v.20.0 (Statistical Package for the Social Sciences). Na sequência, foi realizada uma análise bivariada, para identificar e caracterizar relações entre as variáveis; e foi feita a verificação da normalidade dos dados utilizando-se o teste de correlação do Qui-quadrado ou teste exato de Fischer. Após, foi realizada análise por regressão logística para verificar a existência de fatores confundidores. Para todos os cálculos foi fixado o nível de significância de $95 \%(\mathrm{p} \leq 0,05)$.

\section{Resultados}

Nos dados relacionados, às características das mães dos recémnascidos (Tabela 1), observa-se um risco quase três vezes maior para o parto cesariana $(\mathrm{OR}=2,66) ; 66,7 \%$ das mães dos recém-nascidos com HPIV e 50\% daquelas cujos prematuros não apresentaram HPIV não realizaram pré-natal. Quanto ao nível socioeconômico, 57,1\% das mães do grupo HIPV e 78,8\% do grupo sem HPIV pertenciam às classes A e B.

Os fatores de risco significativamente associados à HPIV (Tabela 2) foram: idade gestacional $(\mathrm{OR}=16,5$; $p<0,001)$, peso ao nascer $(\mathrm{OR}=9,23$; $p<0,01)$ e as práticas neonatais $(\mathrm{OR}=6,68 ; p<0,01)$. Prematuros com idade gestacional $\leq 32$ semanas apresentaram 16,5 vezes mais risco de ter HPIV, sendo este o fator mais fortemente associado com a ocorrência do agravo. O segundo fator de risco foi o baixo peso ao nascer, com razão de chances de ocorrer de 9,23, seguido das práticas neonatais que apresentaram um risco 6,68 vezes maior de serem acometidos pela HPIV. Encontrou-se risco aumentado para a HIPV, de quase três vezes mais, para as crianças que tiveram alguma complicação durante o parto $(\mathrm{OR}=2,66 ; p>0,05)$ e para aquelas cujo Apgar foi menor que $7(\mathrm{OR}=2,66 ; p>0,05)$, embora sem apresentar significância estatística.

Do total das crianças avaliadas, $85,7 \%$ do grupo HPIV e 81,8\% do grupo sem HPIV possuíam até 24 meses de idade. Segundo Papile et al. ${ }^{7}$, $100 \%$ dos prematuros com HPIV foram classificados como HPIV graus I e II (leve). Em relação às características pós-natais dos recém-nascidos, apesar de não terem alcançado significância estatística, observou-se risco de quase o dobro, em crianças que tinham alguma doença neonatal $(\mathrm{OR}=1,79 ; p>0,05)$ e naquelas que tiveram o desenvolvimento neuropsicomotor (DNPM) alterado $(\mathrm{OR}=1,81 ; p>0,05)$ (Tabela 3$)$.

Por meio da análise de regressão logística em relação às variáveis de interesse, obteve-

Tabela 1: Características das mães dos recém-nascidos pré-termo de alto risco associadas à hemorragia peri intraventricular

\begin{tabular}{|c|c|c|c|c|c|c|c|}
\hline \multirow{2}{*}{ Variável } & \multicolumn{2}{|c|}{ Com HPIV } & \multicolumn{2}{|c|}{ Sem HPIV } & \multirow{2}{*}{ OR } & \multirow{2}{*}{ IC (95\%) } & \multirow{2}{*}{$\begin{array}{l}\text { Valor } \\
\text { de } p^{*}\end{array}$} \\
\hline & $\mathrm{n}$ & $\%$ & $\mathrm{n}$ & $\%$ & & & \\
\hline \multicolumn{8}{|l|}{ Tipo de parto } \\
\hline Cesariana & 7 & 50,0 & 9 & 27,3 & 2,66 & $|0,72-9,76|$ & 0,18 \\
\hline Vaginal & 7 & 50,0 & 24 & 72,7 & 1,00 & - & \\
\hline \multicolumn{8}{|l|}{ Pré-Natal } \\
\hline Não & 8 & 66,7 & 14 & 50,0 & 2,00 & $|0,48-8,19|$ & 0,49 \\
\hline Sim & 4 & 33,3 & 14 & 50,0 & 1,00 & - & - \\
\hline \multicolumn{8}{|c|}{ Comorbidades Maternas } \\
\hline Sim & 10 & 71,4 & 21 & 63,6 & 1,42 & $|0,36-5,56|$ & 0,74 \\
\hline Não & 4 & 28,6 & 12 & 36,4 & 1,00 & - & \\
\hline \multicolumn{8}{|c|}{ Nível Sócio Econômico } \\
\hline Classe A e B & 8 & 57,1 & 26 & 78,8 & 0,35 & $0,09-1,38$ & 0,16 \\
\hline Classes $C, D$ e $E$ & 6 & 42,9 & 7 & 21,2 & 1,00 & - & \\
\hline
\end{tabular}

Valor de $p^{*}$ : Teste exato de Fischer.

Fonte: Dados dos autores. 
Tabela 2: Características perinatais associadas ás hemorragia peri-intraventricular em recém-nascidos pré-termo de alto risco

\begin{tabular}{|c|c|c|c|c|c|c|c|}
\hline \multirow{2}{*}{ Variável } & \multicolumn{2}{|c|}{ Com HPIV } & \multicolumn{2}{|c|}{ Sem HPIV } & \multirow{2}{*}{ OR } & \multirow{2}{*}{ IC (95\%) } & \multirow{2}{*}{$\begin{array}{l}\text { Valor } \\
\text { de } p^{*}\end{array}$} \\
\hline & $\mathrm{n}$ & $\%$ & $\mathrm{n}$ & $\%$ & & & \\
\hline \multicolumn{8}{|c|}{ Sexo } \\
\hline Masculino & 9 & 64,3 & 18 & 54,5 & 1,50 & $0,41-5,45$ & 0,74 \\
\hline Feminino & 5 & 35,7 & 15 & 45,5 & 1,00 & - & \\
\hline \multicolumn{8}{|c|}{ Idade Gestacional } \\
\hline$\leq 32$ semanas & 11 & 78,6 & 6 & 18,2 & 16,50 & $3,49-77,98$ & $<0,001$ \\
\hline 33-37 semanas & 3 & 21,4 & 27 & 81,8 & 1,00 & - & \\
\hline \multicolumn{8}{|c|}{ Peso ao nascer } \\
\hline$\leq 1.500 \mathrm{~g}$ & 12 & 85,7 & 13 & 39,4 & 9,23 & $1,77-48,15$ & $<0,01$ \\
\hline$>1.500 \mathrm{~g}$ & 2 & 14,3 & 20 & 60,6 & 1,00 & - & \\
\hline \multicolumn{8}{|c|}{ Complicações no parto } \\
\hline Presentes & 8 & 57,1 & 11 & 33,3 & 2,66 & $0,74-9,61$ & 0,11 \\
\hline Ausentes & 6 & 42,9 & 22 & 66,7 & 1,00 & - & \\
\hline \multicolumn{8}{|c|}{ Apgar no $1^{\circ}$ minuto } \\
\hline$<7$ & 7 & 50,0 & 9 & 27,3 & 2,66 & $0,72-9,76$ & 0,18 \\
\hline$\geq 7$ & 7 & 50,0 & 24 & 72,7 & 1,00 & - & \\
\hline \multicolumn{8}{|c|}{ Práticas neonatais } \\
\hline Presentes & 9 & 64,3 & 7 & 21,2 & 6,68 & $1,69-26,45$ & $<0,01$ \\
\hline Ausentes & 5 & 35,7 & 26 & 78,8 & 1,00 & - & \\
\hline
\end{tabular}

Valor de $\mathrm{p}^{*}$ : Teste exato de Fischer.

Fonte: Dados dos autores.

Tabela 3: Características pós-natais associadas à hemorragia peri-intraventricular em recém-nascidos pré-termo de alto risco

\begin{tabular}{c|c|c|c|c|c|c|c}
\hline Variável & \multicolumn{2}{|c|}{ Com HPIV } & \multicolumn{2}{c|}{ Sem HPIV } & OR & IC (95\%) & $\begin{array}{c}\text { Valor } \\
\text { de } p^{*}\end{array}$ \\
\hline $\begin{array}{c}\text { Doenças Neonatais } \\
\text { Sim }\end{array}$ & 13 & 92,9 & 29 & 87,9 & 1,79 & $0,18-17,65$ & $>0,05$ \\
Não & 1 & 7,1 & 4 & 12,1 & 1,00 & - & \\
\hline DNPM & \\
Alterado & 8 & 57,1 & 14 & 42,4 & 1,81 & $0,51-6,40$ & 0,52 \\
Normal & 6 & 42,9 & 19 & 57,6 & 1,00 & - & \\
\hline Idade no teste \\
0-24 meses
\end{tabular}

Valor de $p^{*}$ : Teste exato de Fischer. DNPM, Desenvolvimento Neuropsicomotor.

Fonte: Dados dos autores.

se que a idade gestacional $(\mathrm{OR}=5,75)$, o peso ao nascer $(\mathrm{OR}=3,60)$ e as práticas associadas $(\mathrm{OR}=4,72)$ apresentaram risco maior que três vezes nas crianças com a doença em estudo, po- rém não alcançaram significância estatística. Em relação aos atrasos no desenvolvimento neuropsicomotor $(\mathrm{OR}=1,11)$ foi encontrado um risco aumentado e igualmente sem significância estatística.

\section{Discussão}

No atual estudo, encontrou-se associação entre a idade gestacional e a hemorragia peri-intraventricular, tornando-se este o principal fator de risco para a doença. Ahn et al. ${ }^{16}$ citam que diferentes estudos ressaltam a evidente relação entre a IG e a incidência de HPIV em recém-nascidos pré-termo. $\mathrm{O}$ estudo de Marret et al. ${ }^{17}$ corrobora estes achados e traz o nascimento prematuro, principalmente naquelas crianças com menor idade gestacional, como um risco elevado para a ocorrência da HPIV e deficit neurológico.

Os resultados igualmente mostraram risco elevado de HPIV em recém-nascidos com muito baixo peso ao nascer (MBPN). Estudos sobre incidência de HPIV em indivíduos recém-nascidos e a relação com o peso ao nascer mostram que a incidência de HPIV varia inversamente naqueles com $\mathrm{MBPN}^{11,16}$. A análise da casuística de uma unidade de terapia intensiva (UTI) neonatal e hemorragia intracraniana/intraventricular evidenciou que essa comorbidade afetou 15 recémnascidos de $\operatorname{MBPN}(4,5 \%)^{23}$. Moreira et al. ${ }^{24}$ também observaram que quase um quarto dos recém-nascidos apresentou alguma morbidade, com maior prevalência nos nascidos com MBPN, e afirmaram que quanto menor o peso ao nascer maior o risco de 
comorbidades, entre elas, a HPIV. Estes resultados confirmam os achados deste estudo, tendo em vista que quanto menor a idade gestacional e também o peso ao nascer, maior tende a ser o risco de ocorrência da HPIV.

Observou-se também, nesta investigação, que as práticas de assistência neonatal, principalmente as manobras de reanimação, mostraram-se associadas à HPIV. Da mesma forma, foi verificada esta associação em estudo de Mello et al. ${ }^{23}$ sobre morbidade neurológica, entre elas a HPIV, no qual $44 \%$ de recém-nascidos precisaram de ventilação mecânica; e $82 \%$, de reanimação ao nascimento. Araújo et al. ${ }^{15}$ discutem, além disso, a correta indicação e o manejo adequado das técnicas de reanimação em relação aos desfechos neurológicos futuros.

Após a análise multivariada, nenhuma das variáveis isoladamente mostrou significância estatística, possivelmente este fato esteja relacionado às características da amostra (recém-nascidos prematuros, de alto risco, que tinham baixa idade gestacional, baixo peso ao nascer e submetidos a práticas neonatais). Embora não tenham mostrado significância em relação ao risco em si de causarem HPIV isoladamente, em conjunto elas se relacionam. Isso demonstra o que já foi observado em diferentes estudos, nos quais se afirma que o conjunto dessas características, além de outras variáveis perinatais, como a presença de comorbidades, está claramente associado entre si $5,10,12,16,23,24$.

Os dados do estudo atual foram consonantes aos da pesquisa de Araújo et al. ${ }^{15}$, que demonstraram que, quando avaliados os fatores associados ao atraso no desempenho motor de recém-nascidos, quase um quarto deles apresentaram alguma morbidade e que quanto menor a idade gestacional, maior o risco de apresentar atraso motor, chegando a encontrar $66,7 \%$ de crianças com alterações. Outras pesquisas 1,15,24-26 apontaram que quanto menor o peso ao nascer maior o risco de comorbidades, com alterações motoras e comportamentais. Na amostra estudada, não foi detectada a ocorrência de atraso no DNPM com significância estatística em prematuros de alto risco com e sem HPIV. Entretanto, a combinação desses fatores de risco relacionados à HPIV, como os encontrados aqui, é considerada fonte de preocupação na literatura recente ${ }^{1,15,24-26}$.

Neste estudo, metade dos recém-nascidos com HPIV nasceram por cesariana. Comparados aos que nasceram de parto vaginal, os recémnascidos pré-termo de alto risco submetidos ao parto cesariano apresentaram mais que o dobro de chances $(\mathrm{OR}=2,66)$ de desenvolver HPIV, ainda que sem diferença estatística. No estudo de Nitsche et al. ${ }^{27}, 80,95 \%$ das crianças com HPIV também foram submetidas ao parto do tipo cesárea. Mello et al. ${ }^{23}$ apresentam também em seus resultados que a maior parte dos partos nas crianças nascidas com idade gestacional $\leq 32$ semanas e/ou peso inferior a $1500 \mathrm{~g}$ ocorreu por cesariana $(82 \%)$.

Os recém-nascidos que tiveram alguma complicação no parto e aqueles com Apgar $<7$ apresentaram risco aumentado para HPIV, ainda que sem diferença estatística. Em uma investigação realizada com crianças nascidas com IG inferior a 32 semanas e/ou peso inferior a 1500 g, cita-se que a percentagem de crianças com índice Apgar ao $1^{\circ}$ minuto $\leq 7$ foi superior a daquelas com deficit neurosensoriais ${ }^{23}$.

Observa-se na literatura que, nos recémnascidos com HPIV severa (III e IV), quase $60 \%{ }^{15}$ apresentam atraso no desempenho motor ${ }^{15,16} \mathrm{e}$ dados do ultrassom transfontanelar destes pacientes ${ }^{7,12}$ corroboram os achados futuros de alteração motora $^{12,28}$. O que pode justificar os resultados obtidos na avaliação do desenvolvimento motor nesta pesquisa, já que, na amostra estudada, tiveram-se apenas prematuros com HPIV grau I e II. Deve-se ressaltar, apesar disso, a importância do acompanhamento do desenvolvimento destes recém-nascidos, mesmo para os que não apresentam esta morbidade, visto que alterações no DNPM podem ocorrer associadas a outros fatores de risco da prematuridade que, por sua vez, também estão associados à HPIV de graus leves (I e II $)^{15}$. Corroborando esta informação, um estudo australiano de coorte ${ }^{12}$, em que se in- 
vestigaram desfechos do neurodesenvolvimento em 2.414 pré-termos extremos com graus I e II de HPIV, mostrou que estes apresentaram aumento das taxas de danos neurossensoriais, atraso no desenvolvimento, paralisia cerebral e surdez aos 2 e 3 anos de idade corrigida. Neste estudo, graus mais leves de HPIV não divergiram significativamente quanto ao DNPM de recém-nascidos de alto risco com outras doenças neonatais.

No estudo de revisão sobre o efeito do nascimento prematuro no DNPM de Moreira et al. ${ }^{24}$, esses autores destacam que, dos trabalhos levantados nos quais se investigaram a hemorragia intraventricular, apenas em um se encontrou associação com pior desempenho motor. Foi observado que quanto menor a idade gestacional e o peso ao nascimento maior é o risco de alterações de comportamento ${ }^{17}$ e que as modificações de fatores de risco ambientais e socioeconômicos ${ }^{29}$ podem melhorar a condição de saúde de crianças nascidas prematuras. Situação esta identificada neste estudo, o que sugere que um nível socioeconômico elevado pode ser um fator protetor para a HPIV.

$\mathrm{O}$ nascimento prematuro constitui-se em um grave problema na área de Saúde da Criança e é reconhecido como fator de risco para atrasos no desenvolvimento infantil. Mesmo com o avanço da tecnologia, voltada para essa área médica, os efeitos da prematuridade ao longo da vida são pouco conhecidos e necessitam de estudos contínuos. Avanços científicos reforçam a possibilidade de evolução de crianças com prognóstico de alterações no desenvolvimento a qual tem sido otimizada pelo diagnóstico e pela intervenção precoce.

Como limitação deste trabalho, destaca-se que a avaliação do DNPM das crianças prematuras com e sem HPIV não foi realizada logo após o nascimento, sendo possível que tenham adquirido suas habilidades tardiamente e fazendo com que os resultados não sejam os mesmos para lactentes. Ainda, todas as crianças avaliadas são recém-nascidas de alto risco com a presença de outras doenças neonatais, o que pode ter gerado similaridade entre os grupos.

\section{Conclusão}

Após análise dos achados desta pesquisa, conclui-se que a baixa idade gestacional e o baixo peso ao nascer estão associados à ocorrência de HPIV e que não existe associação entre HPIV e possíveis atrasos no DNPM.

\section{Agradecimentos}

Os autores agradecem às crianças e suas mães a confiança e disponibilidade para participação neste estudo e ao Hospital Infantil Joana de Gusmão a colaboração para realização desta pesquisa.

\section{Referências:}

1. Poryo M, Boeckh JC, Gortner L, Zemlin M, Duppré P, Ebrahimi-Fakhari D, et al. Ante-, peri- and postnatal factors associated with intraventricular hemorrhage in very premature infants. Early Hum Dev [Internet]. 2017 Jan;116:1-8. Available from: https://linkinghub. elsevier.com/retrieve/pii/S0378378217303572

2. Eisenhut M, Choudhury S. In Premature Newborns Intraventricular Hemorrhage Causes Cerebral Vasospasm and Associated Neurodisability via Heme-Induced Inflammasome-Mediated Interleukin-1 Production and Nitric Oxide Depletion. Front Neurol [Internet]. 2017;8:423. Available from: http://www.ncbi.nlm.nih.gov/pubmed/28868047

3. Mann PC, Woodrum DE, Wilfond BS. Fuzzy Images: Ethical Implications of Using Routine Neuroimaging in Premature Neonates to Predict Neurologic Outcomes. J Pediatr [Internet]. 2013 Aug;163(2):58792. Available from: http://linkinghub.elsevier.com/ retrieve/pii/S0022347613003740

4. Migoto MT, Freire MHS, Barros APMM. Risk factors for perinatal mortality: integrative review. J Nurs Heal. 2018;8(1):1-14.

5. El-Atawi K, Elhalik M, Kulkarni T, Abdelsamed A, Alexander L SA. Risk Factors, Diagnosis, and Current Practices in the Management of Intraventricular Hemorrhage in Preterm Infants: A Review. Acad J Ped Neonatol. 2016;1(3):1-7. 
6. WHO. International classification of impairments, disabilities and handicaps. Geneve [Internet]. 1980;1-207. Available from: https://apps.who.int/ iris/bitstream/handle/10665/41003/9241541261_eng. pdf;jsessionid=D510B46D82787F7C37814FB31C02F98 $\mathrm{F}$ ? sequence $=1$

7. Papile LA, Burstein J, Burstein R, Koffler H. Incidence and evolution of subependymal and intraventricular hemorrhage: a study of infants with birth weights less than 1,500 gm. J Pediatr [Internet]. 1978 Apr;92(4):529-34. Available from: http://www. ncbi.nlm.nih.gov/pubmed/305471

8. Dekom S, Vachhani A, Patel K, Barton L, Ramanathan R, Noori S. Initial hematocrit values after birth and peri/intraventricular hemorrhage in extremely low birth weight infants. J Perineonatology. 2018;38:1471-5.

9. Kavas N, Arısoy AE, Bayhan A, Kara B, Günlemez A, Türker G, et al. Neonatal sepsis and simple minor neurological dysfunction. Pediatr Int [Internet]. 2017 May;59(5):564-9. Available from: http://doi.wiley. com/10.1111/ped.13217

10. Huvanandana J, Nguyen C, Thamrin C, Tracy M, Hinder M, McEwan AL. Prediction of intraventricular haemorrhage in preterm infants using time series analysis of blood pressure and respiratory signals. Sci Rep [Internet]. 2017 Apr 24;7:46538. Available from: http://www.ncbi.nlm.nih. gov/pubmed/28436467

11. Radic JAE, Vincer M, McNeely PD. Outcomes of intraventricular hemorrhage and posthemorrhagic hydrocephalus in a population-based cohort of very preterm infants born to residents of Nova Scotia from 1993 to 2010. J Neurosurg Pediatr [Internet]. 2015 Jun;15(6):580-8. Available from: https://thejns. org/view/journals/j-neurosurg-pediatr/15/6/ article-p580.xml

12. Bolisetty S, Dhawan A, Abdel-Latif M, Bajuk B, Stack J, Lui K. Intraventricular Hemorrhage and Neurodevelopmental Outcomes in Extreme Preterm Infants. Pediatrics [Internet]. 2014;133(1):1-10. Available from: https://pediatrics.aappublications. org/content/pediatrics/133/1/55.full.pdf

13. Apgar V. Evaluation of the newborn infantsecond report. J Am Med Assoc [Internet]. 1958 Dec 13;168(15):1985. Available from: http://jama. jamanetwork.com/article.aspx?doi=10.1001/ jama.1958.03000150027007
14. De Jesus LC, Pappas A, Shankaran S, Li L, Das A, Bell EF, et al. Outcomes of small for gestational age infants born at $<27$ weeks' gestation. J Pediatr [Internet]. 2013 Jul;163(1):55-60.e1-3. Available from: http://www.ncbi.nlm.nih.gov/pubmed/23415614

15. Araújo AT da C, Eickmann SH, Coutinho SB. Fatores associados ao atraso do desenvolvimento motor de crianças prematuras internadas em unidade de neonatologia. Rev Bras Saúde Matern Infant [Internet]. 2013 Jun;13(2):119-28. Available from: http://www.scielo.br/scielo.php?script=sci arttext\&pid=S1519-38292013000200005\&lng=pt\&tlng $=\mathrm{pt}$

16. Ahn SY, Shim S-Y, Sung IK. Intraventricular Hemorrhage and Post Hemorrhagic Hydrocephalus among Very-Low-Birth-Weight Infants in Korea. J Korean Med Sci [Internet]. 2015;30(Suppl 1):S52. Available from: http://synapse.koreamed.org/DOIx. php?id=10.3346/jkms.2015.30.S1.S52

17. Marret S, Marchand-Martin L, Picaud J-C, Hascoët J-M, Arnaud C, Rozé J-C, et al. Brain Injury in Very Preterm Children and Neurosensory and Cognitive Disabilities during Childhood: The EPIPAGE Cohort Study. Wang K, editor. PLoS One [Internet]. 2013 May 2;8(5):e62683. Available from: http://dx.plos. org/10.1371/journal.pone.0062683

18. ABEP. Critério de Classificação Econômica Brasil [Internet]. Critério de Classificação Econômica Brasil. 2012. p. 1-5. Available from: http://www. abep.org/criterio-brasil

19. Frankenburg KW, Dodds J, Archer P, Bresnick B. Denver II: Technical Manual and Training Manual. Denver, USA: Denver Developmental Materials Inc.; 1990.

20. Figueiras AC, Souza ICN, Rios VG, Benguigui Y. Manual Para Vigilância Do Desenvolvimento Infantil Manual Para Vigilância Do. Organ PanAmericana Saúde [Internet]. 2005; Available from: http://www.bvsde.paho.org/bvsacd/cd61/ vigilancia.pdf

21. Halpern R, Figueiras ACM. Influências ambientais na saúde mental da criança. J Pediatr (Rio J) [Internet]. 2004 Apr;80(2):104-10. Available from: http://www.scielo.br/scielo.php?script=sci_ arttext\&pid=S0021-75572004000300013\&lng=pt\&nrm $=\mathrm{iso} \& \mathrm{t} \operatorname{lng}=\mathrm{pt}$

22. Frankenburg WK, Dodds J, Archer P, Shapiro H, Bresnick B. The Denver II: a major revision and restandardization of the Denver Developmental Screening Test. Pediatrics [Internet]. 1992 Jan;89(1):91-7. Available from: http://www.ncbi.nlm. nih.gov/pubmed/1370185 
23. Mello GL, Cristovam MAS, Câmara JPP, Plewka ACL, Ciupak LF. Morbimortalidade entre recémnascidos de muito baixo peso: casuística de uma unidade de terapia intensiva neonatal. Rev Ped Mod. 2014;50(11):203-8.

24. Moreira RS, Magalhães LC, Alves CRL. Effect of preterm birth on motor development, behavior, and school performance of school-age children: a systematic review. J Pediatr (Rio J) [Internet]. 2014;90(2):119-34. Available from: http://www.ncbi. nlm.nih.gov/pubmed/24370176

25. Figueiró-Filho EA, Oliveira VM, Ferreira CM, Silva VM, Tinos AL da S, Kanomata LB. Variáveis perinatais e associação de recém-nascidos de muito baixo peso ao nascer em hospital público universitário do Brasil. Rev Bras Ginecol Obs. 2014;36(1):10-6.

26. Van Hus JW, Potharst ES, Jeukens-Visser M, Kok JH, Van Wassenaer-Leemhuis AG. Motor impairment in very preterm-born children: links with other developmental deficits at 5 years of age. Dev Med Child Neurol [Internet]. 2014 Jun;56(6):587-94. Available from: http://doi.wiley.com/10.1111/ dmcn.12295
27. Nitsche A, Kliemann R, Manfrim EB, Zeigelboin BS, Liberalesso P. Hemorragia cerebral em recémnascidos de baixo peso e o uso de sulfato de magnésio pré-natal. Rev Ped Mod. 2014;50(2):65-8.

28. Hintz SR, Barnes PD, Bulas D, Slovis TL, Finer NN, Wrage LA, et al. Neuroimaging and Neurodevelopmental Outcome in Extremely Preterm Infants. Pediatrics [Internet]. 2015 Jan 1;135(1):e32-42. Available from: http://pediatrics.aappublications. org/cgi/doi/10.1542/peds.2014-0898

29. Khandaker GM, Stochl J, Zammit S, Lewis G, Jones PB. A population-based longitudinal study of childhood neurodevelopmental disorders, IQ and subsequent risk of psychotic experiences in adolescence. Psychol Med. 2014 Nov;44(15):3229-38. 